\title{
SIMPLE QUANTITATIVE ANALYSIS OF SPATIO-TEMPORAL DIAGRAMS FOR DOMAIN WALL DYNAMICS
}

\author{
R.A. KosińSKI AND R. STĘPIEŃ \\ Institute of Physics, Warsaw University of Technology \\ Koszykowa 75, 00-662 Warszawa, Poland
}

(Received September 5, 1994; revised version December 12, 1994)

\begin{abstract}
A quantitative analysis of spatio-temporal diagrams, which describe the dynamics of a chosen, spatially extended dynamic system (a domain wall in a thin magnetic garnet film) is presented. Two quantifiers characterizing such diagrams - a correlation function and power spectrum density function were calculated using solutions of equations of motion. It was found that introducing these quantifiers to the analysis of spatio-temporal diagrams enables one to effectively characterize the type and the properties of motion of the considered system.
\end{abstract}

PACS numbers: 05.45.+b, 75.10.Hk, 75.60.Ch

\section{Introduction}

Recently one can observe that spatio-temporal diagrams play an important role in the investigations of dynamics of spatially extended nonlinear systems [1]. A number of works in this field was devoted to the dynamics of the systems which can be described by simple coupled maps [2-4]. A spatio-temporal diagram technique was also applied successfully to the examinations of collective phenomena such as thermal convection [5,6], boiling of liquids [7] or neuron systems [8]. In this approach quantifiers characterizing diagrams such as pattern entropy or Lyapunow exponents, were introduced in order to give the synthetic information about the dynamics of the system $[1,3,9]$.

In the present work other quantifiers characterizing spatio-temporal diagrams - a correlation function and power spectrum density function - were used. As a nonlinear, spatially extended system a domain wall in a thin magnetic garnet film, described by partial differential equations of motion, was analyzed. Such systems were extensively investigated experimentally as well as theoretically, mainly due to the applications in magnetic memory devices [10-14]. A short description of its dynamics is given in Sec. 3. 


\section{Equations of motion of domain wall}

A one-dimensional model of a domain wall is considered. In this model the domain wall is represented by a chain of magnetic moments (see Fig. 1), lying at the Bloch surface (i.e. in the middle plane of a static wall [10]) in the middle plane of the garnet film. Equations of motion for such a wall result from

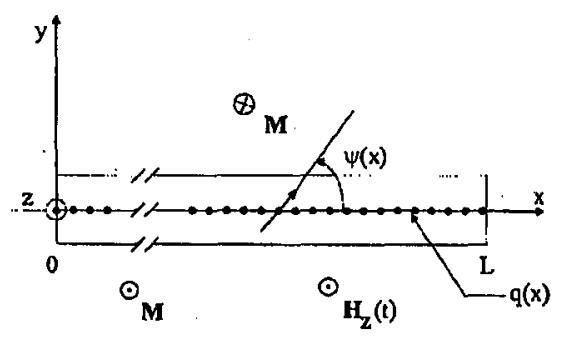

Fig. 1. A model of a domain wall of the length $L ; M$ denotes the magnetization in the domains adjacent to the wall, $\boldsymbol{H}_{z}(t)$ is the magnetic drive field. Time evolution of the azimuthal angle of magnetization $\psi(x)$ and the position of Bloch surface $q(x)$ describe the wall motion.

Landau-Lifschitz-Gilbert equation and have a form which has been proposed by Slonczewski [10]

$$
\begin{aligned}
& \frac{\dot{q}}{\Delta}=2 \pi \gamma M \sin 2\left(\psi-\psi_{t}\right)-\frac{2 A \gamma}{M} \frac{\partial^{2} \psi}{\partial x^{2}}+\alpha \dot{\psi}, \\
& \dot{\psi}=\gamma H_{z}+\frac{2 A \gamma}{M \Delta} \frac{\partial^{2} q}{\partial x^{2}}-2 \pi \gamma M \Delta \frac{\partial}{\partial x}\left[\sin 2\left(\psi-\psi_{t}\right)\right]-\frac{\alpha}{\Delta} \dot{q},
\end{aligned}
$$

where $M$ is the saturation magnetization, $A-$ the exchange constant, $\Delta=$ $\sqrt{A / K_{\mathrm{u}}}$ - the Bloch wall width parameter, $K_{\mathrm{u}}$ - the uniaxial anisotropy constant, $\gamma$ - the gyromagnetic ratio, $\psi_{t}$ - the angle between the $+O x$ axis and the direction tangent to the wall, $\alpha$ - the damping parameter, $H_{z}(t)$ - the external drive field, $q(x, t)$ is the position of the Bloch surface of the wall, $\psi(x, t)$ is the azimuthal angle of $\boldsymbol{M}$ at the Bloch surface. A dot over a symbol denotes the time derivative. In our computations the drive field of the periodic form $H_{z}(t)=H_{0} \sin (2 \pi t / T)$ was chosen. Initial conditions of the form $q(x, 0)=0$, $\psi(x, 0)=0$ and periodic boundary conditions were assumed. Equations $(1,2)$ were solved numerically using the full implicit scheme for the segment of the wall of the length $L=5 \pi \Lambda$ (where $\Lambda=\sqrt{A / 2 \pi M^{2}}$ is the Bloch line width parameter) divided into $N=52$ numerical points with the time step of the integration procedure $\Delta T=0.1 \mathrm{~ns}[15]$.

The material parameters were: $M=11.14 \mathrm{Gs}, A=0.81 \times 10^{-7} \mathrm{erg} / \mathrm{cm}$, $\gamma=1.75 \times 10^{7} 1 / \mathrm{Oe} / \mathrm{s}, \Delta=2.9 \times 10^{-6} \mathrm{~cm}, \Lambda=1.019 \times 10^{-5} \mathrm{~cm}$. As a value of the damping parameter $\alpha=0.005$ was chosen. For these parameters the length $L$ of the considered segment of the wall equals $1.6 \times 10^{-4} \mathrm{~cm}$, while the time scale of the considered system may be approximated by the time of generation of vertical Bloch lines (VBLs) [10] which is of the order of 2-4 ns. 


\section{Domain wall motion in periodic drive fields}

Using Slonczewski's equations of motion [10], it was found that in the case of the periodic drive field three types of motion of the domain wall, periodic, quasiperiodic and chaotic, may occur $[9,16,17]$. All these types of motion are connected with characteristic structures of the wall and their ranges depend on the values of the amplitude $H_{0}$ and the period $T$ of the drive field, as well as on the damping parameter $\alpha[16,17]$.

In the case of the periodic motion, translational oscillations of the wall with the uniform structure $(\psi(x)=$ const, $q(x)=$ const) are observed. The frequency of these oscillations is the same as the drive field frequency $\omega_{0}=2 \pi / T$.

It is also possible that, for certain ranges of the amplitude $H_{0}$, small periodic deformations of the wall structure appear. In the case of $\psi(x)$ they have amplitude smaller than approximately $10^{\circ}$ and, due to the periodic boundary conditions assumed at the terminations of the considered wall segment, may have a form of waves propagating with different velocities along the wall. Certain characteristic frequencies are connected with such waves; they depend on the drive field frequency $\omega_{0}$, and are responsible for the quasiperiodic motion of the wall. Thus, in such a case the translational, oscillatory motion of the wall (as in the periodic case) is superimposed on the oscillations of the wall structure. It is interesting that such oscillations may be generated even at very small values of $H_{0}$, i.e. the quasiperiodic motion occurs also for $H_{0}$ close to zero. This is connected with the fact that for small damping ( $\alpha=0.005$ as used here) the wall structure is very susceptible for deformations. In such a case a part of the energy delivered to the magnetic sample from the drive field is stored in oscillations of the wall structure and a part of it is connected with demagnetizing of the film due to the translational wall motion. For other values of $H_{0}$, for which the periodic motion is observed, the whole energy is connected with the translational oscillations of the wall. These phenomena may be connected with the spin waves, propagating in the magnetic medium $[18,17]$ and they demand the further investigations.

The ranges of the quasiperiodic and periodic motion are interlaced [17] and, when the drive field amplitude is large enough, certain structures of the wall, called vertical Bloch lines pairs (VBLs), appear [10-14]. VBLs are generated and annihilated (their number and angular span change during the wall motion) and move along the wall. Also the surface of the wall is very corrugated. Generally speaking, the complexity of the wall structure increases with increasing amplitude $H_{0}$. In such a case the motion of the wall is chaotic. The lower bound of such a motion is at $H_{0}=H_{0 c r i t}$, which depends on the drive field frequency $\omega_{0}$ and damping parameter $\alpha$. In our case $H_{0 \text { crit }}=12.99$ Oe, while, for instance, for $\alpha=$ 0.1 it was found that $H_{0 \text { crit }}=24.09 \mathrm{Oe}$. It should be stressed that an appearance of VBLs in the wall always accompanies the chaotic motion of the wall [16].

\section{Construction of spatio-temporal diagrams}

The construction of the spatio-temporal diagrams was based on the numerical solutions of Eqs. $(1,2)$, where one of the two variables, $\psi_{i}(t)$, at each numerical 


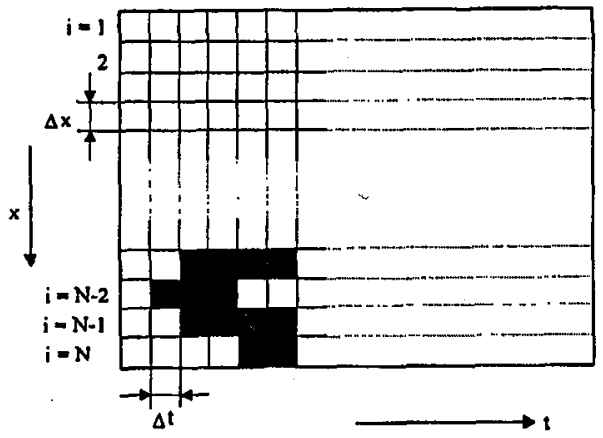

Fig. 2. Spatio-temporal diagrams consist of black or white cells of the size $\Delta x-\Delta t$. Series of -1 and +1 are connected with the color of the cells; for the grid point $i=N-2$ the following series results: $-1,1,1,1,-1,-1 \ldots$
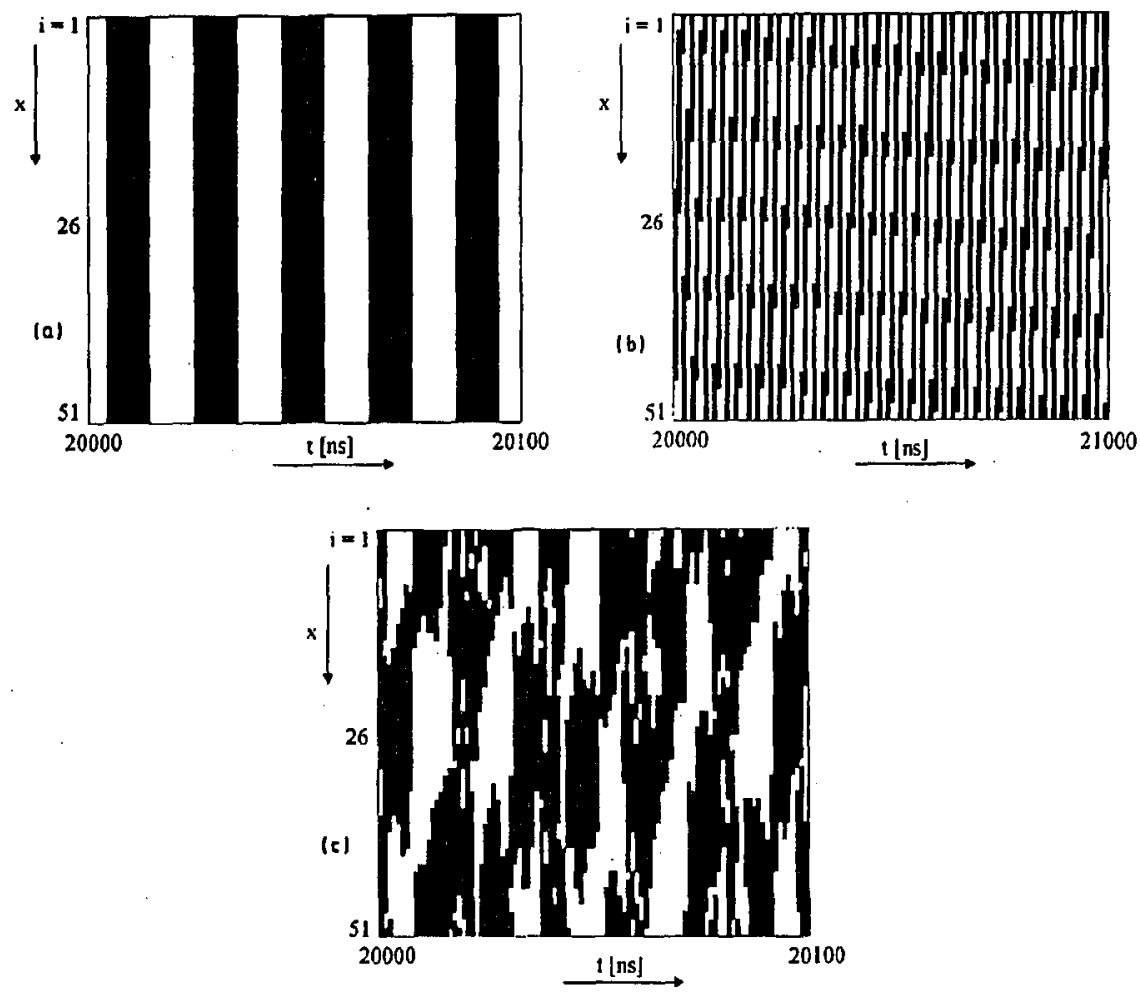

Fig. 3. Spatio-temporal diagrams for three types of the wall motion: (a) the periodic motion for $H_{0}=8 \mathrm{Oe}$, (b) quasiperiodic motion for $H_{0}=2 \mathrm{Oe}$ and (c) chaotic motion for $H_{0}=35 \mathrm{Oe}$.

point $(i=1, \ldots, N)$ was used. The choice of the variable $\psi$ is connected with the fact that in the applications, as well as in a description interesting physical phe- 
nomena, internal structures of the wall are commonly characterized in literature using just $\psi(x, t)$.

The time evolution of $\psi_{i}(t)$ was digitized using the following rule: in each $n$-th time step of the integration procedure the values -1 or +1 were assigned to the $i$-th grid point if $\left|\psi_{i}\right|<|\bar{\psi}+\varepsilon|$ or $\left|\psi_{i}\right| \geq|\bar{\psi}+\varepsilon|$, respectively (where $\varepsilon$ was assumed to be equal $0.001 \mathrm{rd}$ ). $\bar{\psi}$ denotes here the spatial average of $\psi$ over the segment of the wall $L$ (cf. Ref. [9]). Thus, the information contained in the solutions of Eqs. $(1,2)$ was reduced, however as we will see, a type of the wall motion is maintained in the time series of -1 and +1 of each grid point (see Fig. 2), which appeared instead of the sequence of solutions $\psi_{i}(\Delta t=n \Delta T),(n=10,20,30, \ldots)$. The spatio-temporal diagrams for the whole considered segment of the wall were constructed as sets of the sequences of white (for -1$)$ and black $($ for +1$)$ cells of the size $\Delta x=L / N, \Delta t=10 \Delta T$ for all grid points (see Fig. 2). For instance for the wall moving periodically with the uniform structure, i.e. with $\psi(x)=$ const, the spatio-temporal diagram consists of a set of parallel white and black stripes (see Fig. 3a). It should be mentioned that mapping of $\psi_{i}(n \Delta T)$ into the set $\{-1,1\}$ was chosen because it is useful for a power spectrum density analysis. In such mapping however, some information about dynamic processes in the system, for instance the VBLs generation [10], is not seen.

\section{Correlation function for spatio-temporal diagrams}

As a first quantifier synthetically described spatio-temporal diagrams a correlation function was used. It was calculated for two series of -1 and $+1: a(t)$ corresponding to the first $(i=1)$ and $b(t)$ corresponding to the middle $(i=N / 2)$ grid points of the wall, using the definition [19]

$$
C(\tau)=\sum_{n=0}^{L-1} a_{n} b_{n+\tau},
$$

where $a_{n}, b_{n}$ are sampled time signals, $L$ denotes an amount of samples and $\tau$ is the time shift. In the computations of this function fast Fourier transform with a zero padding technique was used [19]. Due to the periodic boundary conditions used in the solution of Eqs. $(1,2)$ the first $(i=1)$ and the middle $(i=N / 2)$ points are located opposite to each other, as it is shown in Fig. 4 . The series of $-1,+1$ were registered during numerical solutions of Eqs. $(1,2)$ for the time $t_{w}=8192 \mathrm{ns,}$ starting at the end of the transient time $t_{\mathrm{tr}}$, which was assumed as $t_{\mathrm{tr}}=10 \mu \mathrm{s}$.

Calculations were performed for the domain wall moving in the periodic, drive field $H_{z}(t)$ with the chosen value of its period $T=20 \mathrm{~ns}$ and the amplitude $H_{0}$ treated as a control parameter. The dynamics of a wall in such a drive was investigated earlier $[9,16]$, however for $T=25 \mathrm{~ns}$.

The correlation functions with the time shift $\tau$ between signals $a(t)$ and $b(t)$ were calculated for three chosen values of $H_{0}$ for which different types of the wall motion occur. Results are shown in Fig. 5, where only one point per period of the drive field (i.e. each 20-th point) of the correlation function is marked to make the plot more clear.

For $H_{0}=8$ Oe the correlation function has a linear form reaching value 1 for $\tau=0 \mathrm{~ns}$ and decreasing to zero for $\tau=t_{w}=8192 \mathrm{~ns}$, (i.e. for $\tau$ equalled the lengths 


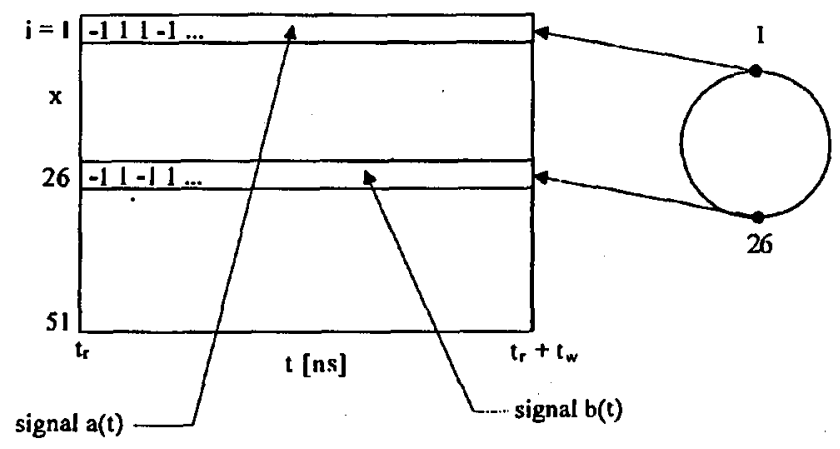

Fig. 4. Sequences of -1 and 1 for which the correlation function was calculated. The discrete signal $a(t)$ comes from the first grid point $(i=1)$, signal $b(t)$ comes from the middle grid point $(i=N / 2) . t_{r}$ is the transient time, $t_{w}$ is the length of the signals $a(t)$ and $b(t)$.
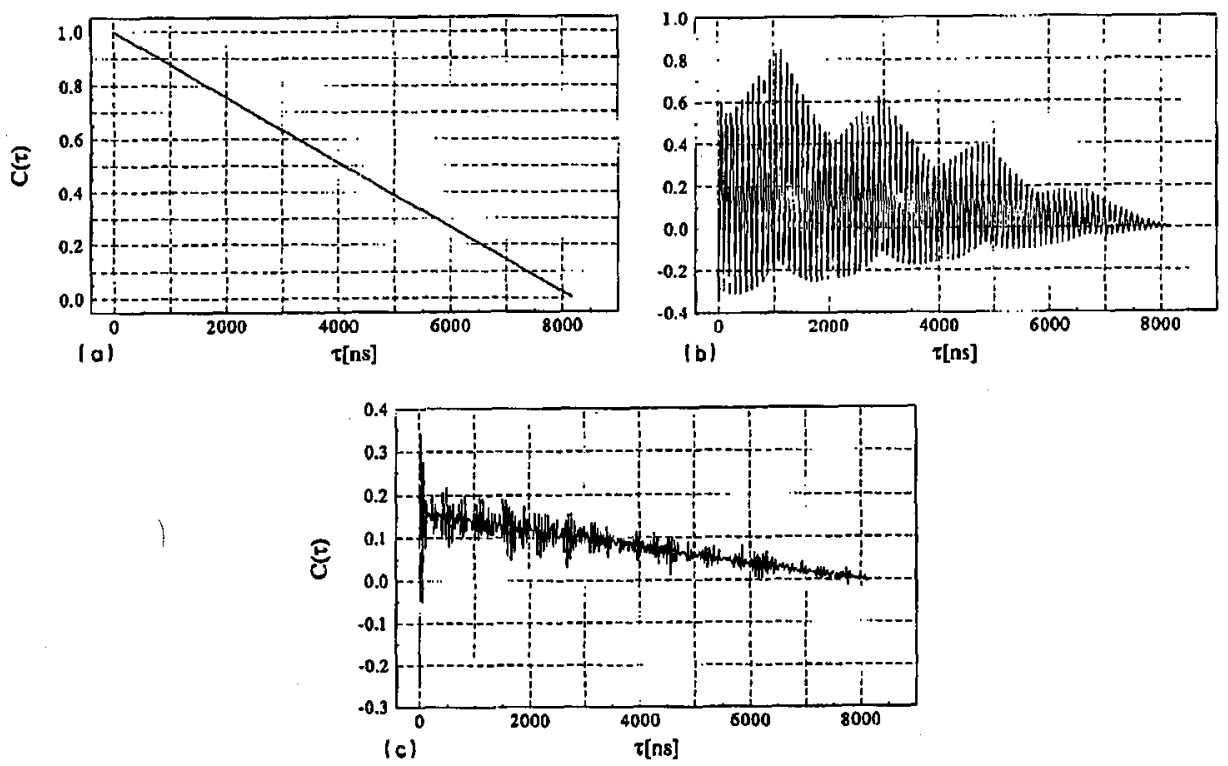

Fig. 5. The correlation function $C$ as a function of a time shift $\tau$ between signals $a(t)$ and $b(t)$ for three types of the wall motion. Only one point per drive field period (each 20-th point) is shown. (a) The periodic motion, (b) quasiperiodic motion, (c) chaotic motion. The values of the drive field amplitude are the same as in Fig. 3(a,b,c), respectively.

of each signal $a(t)$ and $b(t))$. This means that the wall moves periodically and its structure remains uniform (cf. the spatio-temporal diagram for the same amplitude $H_{0}$, Fig. 3a). For $H_{0}=2$ Oe the correlation function oscillates regularly with the mean value tending to 0 for a time shift $\tau=8192 \mathrm{~ns}$ (see Fig. 5b). These oscillations 
are connected with quasiperiodic oscillations of $\psi(x, t)$ which are clearly observed at the spatio-temporal diagram in Fig. 3b (in this figure, however. $\Delta t=10 \mathrm{~ns}$ ). For $H_{0}=35$ Oe irregular oscillations of the correlation function were obtained (Fig. 5c). The time evolution of $\psi(x)$ is chaotic as the spatio-temporal diagram shows (Fig. 3c). The periodic, quasiperiodic and chaotic motion of the domain wall were also found earlier using Poincare section of the phase trajectory in a chosen phase subspace $[14,16]$ and time dependence of pattern entropy [9].

\section{Harmonic analysis of spatio-temporal diagrams}

For the spatio-temporal diagrams under investigations a harmonic analysis was performed. It was based on a calculation of the power spectrum density of the discrete signal $b(t)$, defined as in the previous paragraph, and coming from the middle point of the wall $(i=26)$.
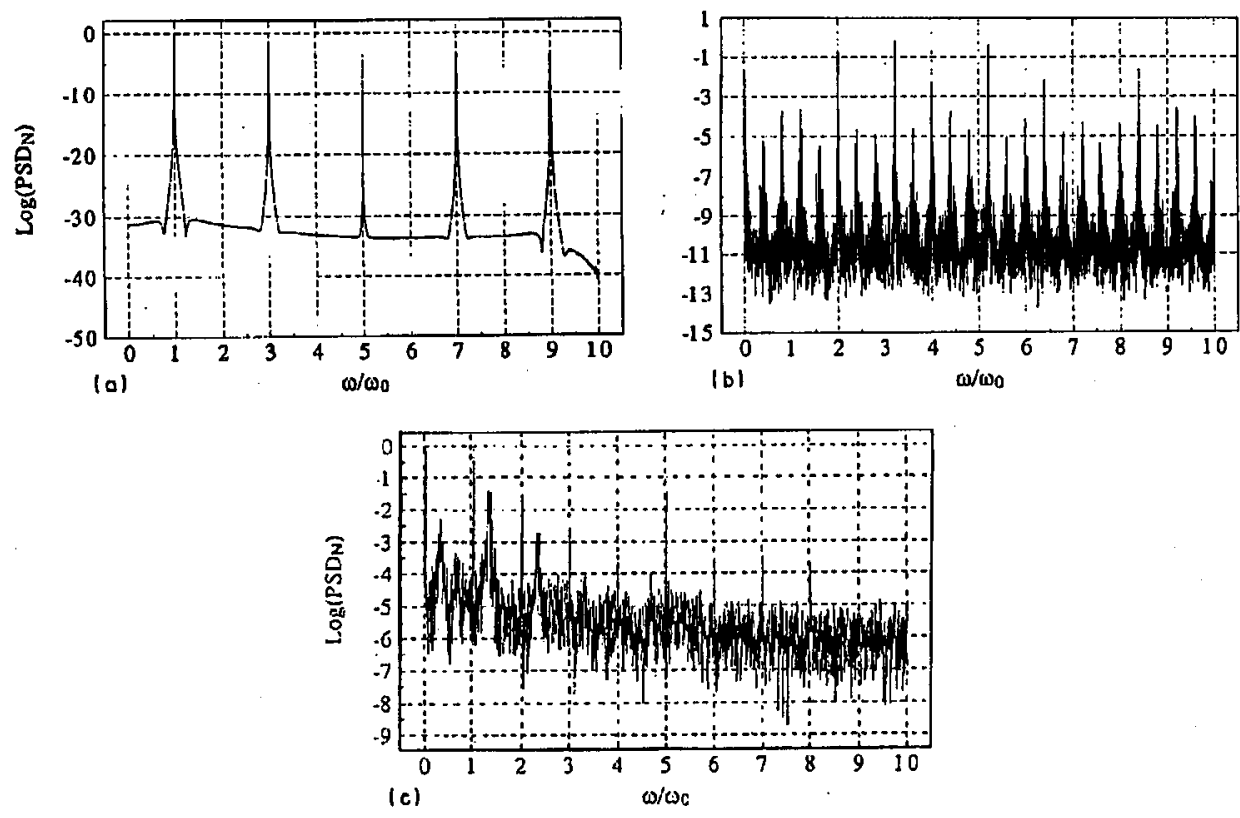

Fig. 6. Logarithm of the relative power spectrum density function $\operatorname{PSD}_{N}$ as a function of relative frequency $\omega / \omega_{0}\left(\omega_{0}\right.$ is the drive field frequency). (a) The periodic wall motion, (b) quasiperiodic wall motion, (c) chaotic wall motion. The values of the drive field amplitude are the same as in Fig. $3(a, b, c)$, respectively.

The power spectrum density $\left(\mathrm{PSD}_{N}\right)$ for three values of the amplitude of the drive field (the same as in the previous section) were estimated by periodogram $[19,20]$. Results are shown in Fig. 6 , where the power spectrum density function was represented in a logarithmic form. For the case of the periodic motion of the wall $\left(H_{0}=8 \mathrm{Oe}\right.$, see Fig. $\left.6 \mathrm{a}\right)$ the first peak of the curve corresponds to the 
frequency of the drive field $\omega_{0}$, while other peaks are harmonics of the $\omega_{0}$ frequency, no other characteristic frequencies are present. For $H_{0}=2$ Oe at the power spectrum density (Fig. 6b) two characteristic frequencies $\omega_{1} \simeq \omega_{0} / 2$ and $\omega_{2} \simeq 0.8 \omega_{0}$ are seen (other peaks are connected with their harmonics). The peak corresponding to the frequency equal to the drive frequency $\omega_{0}$ is not visible, because the ratios of the powers of the signal components connected with the harmonics $\omega_{0}$ and with the harmonics $\omega_{1}$ and $\omega_{2}$ are small. Thus, one can see that the power spectrum density function enables one to define some characteristic frequencies of the quasiperiodic motion of the domain wall. Some of them, however, may be suppressed due to the distribution of power in the signal (as was explained above) or due to the time sampling of the solutions of equations of motion. The chaotic motion of the wall, which occurs for $H_{0}=35 \mathrm{Oe}$, is also clearly visible on the power spectrum density function (Fig. 6c). It decreases as $1 / f$ (where $f \equiv \omega / 2 \pi$ ) and only some unregularly located peaks are observed.

\section{Conclusions}

The frequency analysis of the spatio-temporal diagrams (using the correlation function and power spectrum density function) seems to be an effective tool which enables one to define a type and the properties of motion of the spatially extended dynamical systems. Moreover, such analysis using binary coding is faster and more clear than a frequency analysis of full solutions of equations of motion, because of the redundant information contained in full solutions.

During the computations the fact that the presence of vertical Bloch line pairs accompanies the chaotic motion of the wall $[16,17]$ was confirmed.

\section{Acknowledgments}

The authors wish to thank Dr. T. Tymosz for critical reading of the manuscript of this work.

\section{References}

[1] Formation, Dynamics and Statistics of Patterns, Eds. K. Kawasaki, M. Suzuki, A. Onuki, World Science, Singapore 1990.

[2] G. Mayer-Kress, K. Kaneko, J. Stat. Phys. 54, 1489 (1989).

[3] K. Kaneko, Europhys. Lett. 6, 193 (1988).

[4] D. Stasinopoulos, P. Alstrom, to be published.

[5] T. Yanagita, K. Kaneko, Phys. Lett. A 175, 415 (1993).

[6] F. Dariaud, J. Lega. P. Berge, P. Coullet, M. Dubois, Physica D 55, 287 (1992).

[7] Y. Yanagita, Phys. Lett. A 165, 405 (1993).

[8] W. Wang, G. Perez, H.A. Cerdeira, Phys. Rev. E 47, 2893 (1993).

[9] R. Kosiniski, Phys. Lett. A 169, 263 (1992).

[10] A.P. Malozemoff, J.C. Slonczewski, Magnetic Domain Walls in Bubble Materials, American Press, New York 1979. 
[11] S. Konishi, K. Matsuyama, I. Chida, S. Kubota, H. Kawahara, M. Ohbo, IEEE Trans. Magn. MAG-20, 1129 (1984).

[12] F.B. Humphrey, J.C. Wu, IEEE Trans. Magn. MAG-21, 1762 (1985).

[13] J.J. Żebrowski, Phys. Scr. 38, 632 (1988).

[14] R.A. Kosiński, A. Sukiennicki, J. Magn. Magn. Mater. 104-107, 331 (1991).

[15] R.A. Kosiński, J. Engemann, J. Magn. Magn. Mater. 50, 229 (1985).

[16] R.A. Kosiński, IEEE Trans. Magn. 30, 1233 (1994).

[17] R.A. Kosiński, J. Appl. Phys. 73, 320 (1993).

[18] J.E. Janak, Phys. Rev. 124, 452 (1961).

[19] W.H. Press, B.P. Flannery, S.A. Teukolsky, W.T. Vetterling, Numerical Recipes, Cambridge University Press, Cambridge 1986.

[20] F.J. Harris, IEEE Proc. 66, 51 (1978). 